\title{
Harnessing the damping properties of materials for high-speed atomic force microscopy
}

\author{
Jonathan D. Adams1', Blake W. Erickson', Jonas Grossenbacher², Juergen Brugger², \\ Adrian Nievergelt ${ }^{1}$ and Georg E. Fantner ${ }^{1 \star}$
}

The success of high-speed atomic force microscopy in imaging molecular motors ${ }^{1}$, enzymes ${ }^{2}$ and microbes $^{3}$ in liquid environments suggests that the technique could be of significant value in a variety of areas of nanotechnology. However, the majority of atomic force microscopy experiments are performed in air, and the tapping-mode detection speed of current highspeed cantilevers is an order of magnitude lower in air than in liquids. Traditional approaches to increasing the imaging rate of atomic force microscopy have involved reducing the size of the cantilever 4,5 , but further reductions in size will require a fundamental change in the detection method of the microscope $\mathrm{e}^{6-8}$. Here, we show that high-speed imaging in air can instead be achieved by changing the cantilever material. We use cantilevers fabricated from polymers, which can mimic the high damping environment of liquids. With this approach, SU-8 polymer cantilevers are developed that have an imaging-in-air detection bandwidth that is 19 times faster than those of conventional cantilevers of similar size, resonance frequency and spring constant.

A primary research goal in atomic force microscopy (AFM) is to increase the imaging speed, improve its ease of use and expand its potential range of applications ${ }^{9}$. In the most widely used AFM mode (a.c. mode or tapping mode) the detection speed (mechanical bandwidth, $B W$ ) of the AFM cantilever fundamentally limits the imaging speed. The bandwidth is a measure of the maximum rate of topography change the cantilever can accurately detect. It is related to the cantilever resonance as $B W \propto f_{0} / Q$, where the cantilever resonance frequency $f_{0}$ is primarily determined by the cantilever mass and elastic modulus, and the quality factor $Q$ is determined by the cantilever damping ${ }^{10}$. When the oscillating cantilever experiences a change in boundary condition (that is, topography), it requires several cycles to reach a new steady-state amplitude (Fig. 1a). A cantilever with higher resonance frequency runs through the required number of cycles more quickly, thereby enabling faster imaging (Fig. 1b). The number of required oscillatory cycles is determined by the damping of the cantilever, characterized by $Q$. A cantilever with low resonance frequency and low $Q$ can therefore be equally as fast as a cantilever with high resonance frequency and high $Q$ (compare Fig. $1 b$ and c). From a detection bandwidth perspective, the ideal combination is a high resonance frequency and low quality factor (Fig. 1d).

The development of current high-speed AFM (HS-AFM) technology was enabled by the miniaturization of silicon and silicon nitride (SiN) cantilevers to $x-y$ dimensions below $10 \mu \mathrm{m}$ (the approach taken in Fig. 1b), resulting in cantilevers with megahertz resonance frequencies ${ }^{4,5}$. Modelling and an improved understanding of cantilever behaviour in fluids has greatly benefited this geometric optimization. For nearly all cantilevers, viscous damping in the surrounding medium determines $Q$ (ref. 11). In liquid, viscous damping yields $Q \approx 2-4$, enabling extremely high imaging speeds. Using small cantilevers immersed in fluid, Ando and colleagues ${ }^{5}$ pioneered close to video-rate AFM imaging and thereby established the field of HS-AFM in liquid on biological samples ${ }^{1-3,12-15}$. Although such cantilevers may also have a relatively low $Q$ in air, their low spring constants make them primarily intended for imaging in fluid. In air, higher spring constants are often required to reduce the effects of surface adhesion. This, coupled with the $\sim 50$-fold lower viscosity, yields $Q \approx 200$. This high $Q$ limits the detection bandwidth in air for even the smallest currently available AFM cantilevers with large spring constants to well below what is achievable in liquid (Fig. 1e). Increasing the cantilever bandwidth by further shrinking the cantilever dimensions and boosting $f_{0}$ is not possible. State-of-the-art HS-AFM systems already use cantilevers approaching the optical diffraction limit. A further size reduction would require fundamentally different detection techniques. Techniques such as near-field scattering and thin-metal-film piezoresistive sensing have been proposed ${ }^{6-8}$. However, such small cantilevers suffer from challenges in their implementation and are limited to use on very flat samples because of the minimal chipsample clearance.

In this Letter, we increase the cantilever bandwidth in air in a manner decoupled from the cantilever size. By using internal damping mechanisms in cantilevers made of viscoelastic material, we mimic the high damping environment in fluid, yielding cantilevers with inherently low quality factors in any medium. The resulting fast-responding cantilevers allow for high imaging speeds in air, even on challenging samples (Fig. 1f).

The optimal material maximizes what we call the 'material bandwidth product', which we define as $\eta_{\mathrm{i}} \sqrt{E / \rho}$, because to first order, the cantilever elastic modulus and density influence the resonance frequency as $\sqrt{E / \rho}$ and the materials contribution to $Q$ is the intrinsic loss factor $\eta_{\mathrm{i}}$ (Fig. 2a and Supplementary Section 1). The material bandwidth products of several polymers are roughly three orders of magnitude above those of traditional cantilever materials ( $\mathrm{Si}$ and $\mathrm{SiN}$ ). The polymer SU-8 is particularly promising as a consequence of its high material bandwidth product and ease of fabrication. Indeed, early micromechanical applications of SU-8 were in AFM cantilever fabrication $^{16}$, although not because of the high materials bandwidth product.

We modelled the expected cantilever bandwidth, given by $\pi f_{0} / Q$ (ref. 10), for a range of rectangular geometries for tapping-mode in air use. Figure $2 \mathrm{~b}$ shows that, for any allowable cantilever geometry, $f_{0}$ and spring constant $k$, we can expect a one order of magnitude increase in detection bandwidth by switching the cantilever material to SU-8 (for details see Supplementary Section 2). To test this hypothesis, we fabricated cantilevers from SU-8 that match the specifications of two widely used conventional cantilevers for tapping 
a
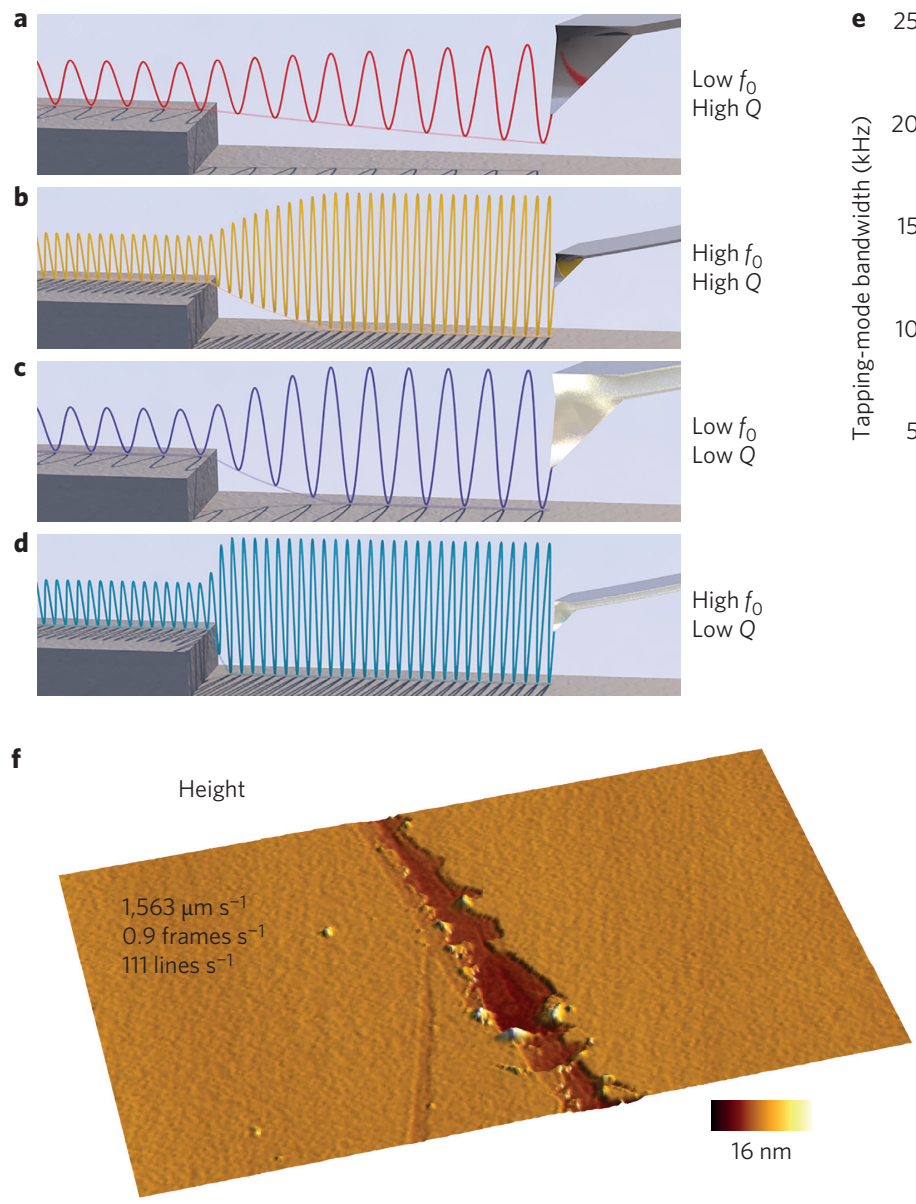

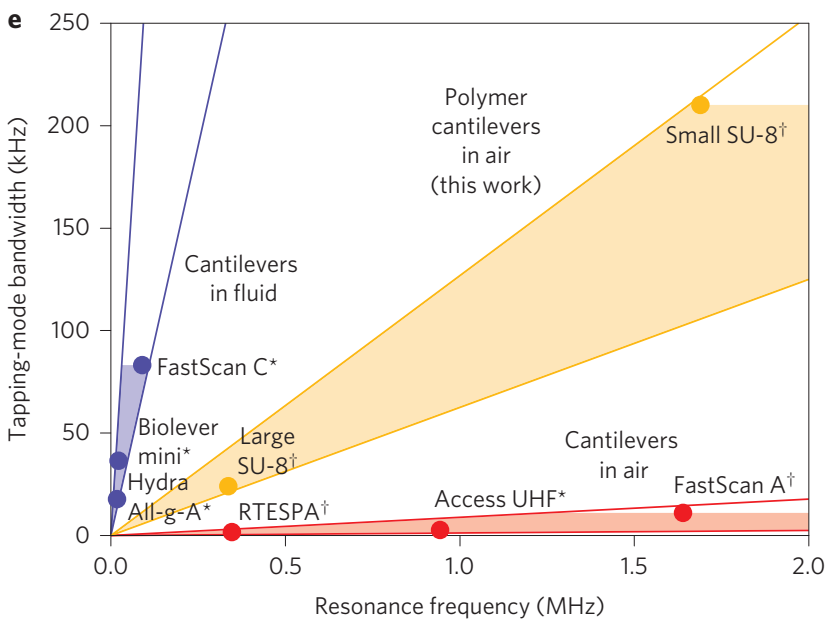

Error
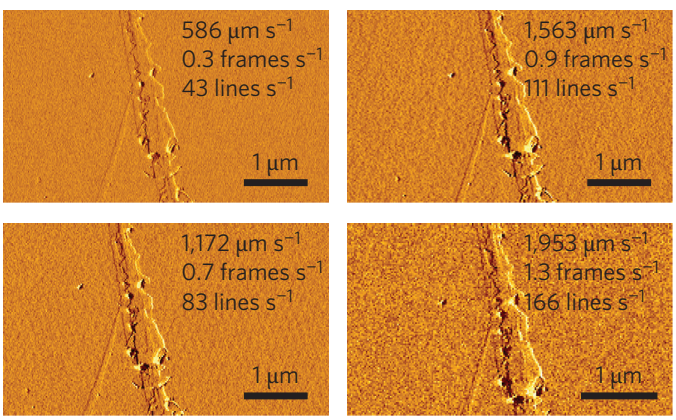

Figure 1 | Why HS-AFM imaging in air has been slower than in fluid. a-d, An oscillating cantilever subject to a boundary condition change has a response time related to the ratio $f_{0} / Q$. Both increasing the resonance frequency $f_{0}(\mathbf{b})$ and decreasing the $Q$ factor (c) lead to similarly improved response times. Using small, high- $f_{0}$, low-Q polymer cantilevers maximizes $f_{0} / Q$ for the fastest cantilever amplitude response in air (d). e, Trends in the development of cantilevers for HS-AFM imaging in fluid and air. Increasing the resonance frequency of a cantilever in fluid strongly increases its imaging bandwidth because the cantilever $Q$ is low in the high damping environment (blue lines). For traditional cantilevers in air, however, an increase in $f_{0}$ only has a marginal influence on the imaging bandwidth (red lines), because the $Q$ remains high in the low damping environment. Using polymers as a cantilever material greatly boosts the cantilever bandwidth in air (yellow lines), because the large internal cantilever damping yields a low $Q$ in any medium. ${ }^{\star}$ Bandwidth predicted from measured values of $f_{0}$ and $Q$ as the ratio $\pi f_{0} / Q$. 'Directly measured bandwidths. The fill level of wedges corresponds to bandwidths achievable on mainstream instruments. f, High-speed tapping-mode imaging in air of sharp step edges in sanded mica. The three-dimensional height image shows a scan rate of $1.5 \mathrm{~mm} \mathrm{~s}^{-1}$ (nearly 1 frame s${ }^{-1}$ ) while maintaining high-quality imaging. The amplitude error images (right) show no evidence of imaging artefacts such as overshoot, parachuting or ringing. Taken together, these images demonstrate good tracking behaviour, even at the highest scan rates. The feedback error images show near-constant tracking quality over a range of scan rates up to 1.3 frames $\mathrm{s}^{-1}$ (the fastest image is pixelated due to controller data rate limitations). Error scale, -5 to $5 \mathrm{~nm}$.

mode imaging in air. We fabricated 'large SU-8' cantilevers to be nominally equal in their $f_{0}, k$ and $x-y$ dimensions to the RTESPA commercial silicon cantilever (Bruker) and 'small SU-8' cantilevers to match the FastScan A commercial small AFM cantilever (Bruker) (Fig. 3). We characterized $f_{0}, k$ and $Q$ from the thermomechanical spectra (Fig. 3b). The resonance frequencies and spring constants matched well within each pair. The resulting $Q$-factor of the large SU-8 cantilever is over an order of magnitude below that of the large Si cantilever (21 versus 350 ), and similar results were found for the small SU-8 cantilever versus the FastScan A (33 versus 180). Finite-element analysis of the independent contributions of air damping and viscoelastic damping confirmed that the $Q$ of the SU- 8 cantilevers is primarily determined by the SU- 8 viscoelasticity (Fig. 3c and Supplementary Sections 3 and 4).

The individual cantilever response times were measured by exciting them at resonance and simulating the topography with a step increase in drive amplitude (Fig. $4 \mathrm{a}$ ). The first $3.5 \mathrm{~ms}$ of the cantilever responses shows the decaying exponential behaviour of the amplitude envelopes. The first $30 \mu$ s shows the individual oscillation cycles. The large Si cantilever with low $f_{0}$ and high $Q$ has the slowest response. Increasing $f_{0}$ speeds up the response (FastScan A), as does decreasing $Q$ (large SU-8), both by an order of magnitude. The high $f_{0}$, low $Q$ cantilever (small SU-8) achieves roughly two orders of magnitude faster amplitude response over the standard cantilever. This faster amplitude response directly translates into a higher tapping-mode bandwidth of the cantilever. Figure $4 \mathrm{~b}$ shows, for each cantilever, the tracking amplitude (vertical axis), which represents the fraction of topography accurately detected by the cantilever, at a given rate of topography change (horizontal axis). We used a $3 \mathrm{~dB}$ decrease in tracking amplitude to define the detection bandwidth. A comparison of the bandwidths of the individual cantilevers (RTESPA, $1.6 \mathrm{kHz}$; FastScan A, $11 \mathrm{kHz}$; large SU-8, $24 \mathrm{kHz}$; small SU-8, $210 \mathrm{kHz}$ ) shows that the gain in bandwidth from a change in material (a factor of 15-19) is higher than the gain in bandwidth achieved by miniaturization (a factor of 7-9). By combining both miniaturization and a change in material we obtained 

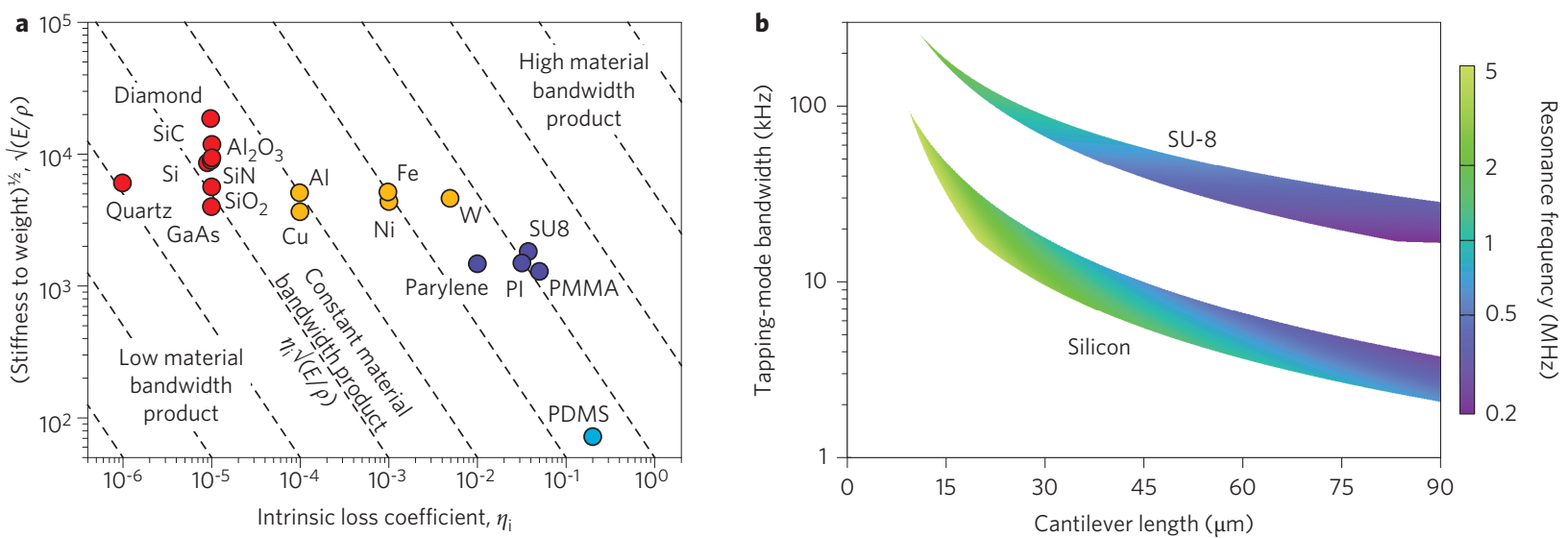

Figure 2 | HS-AFM cantilever design from a materials perspective. a, Potential cantilever materials, classified by their material bandwidth product $\eta_{\mathrm{i}} \sqrt{E / \rho}$ where $E$ is Young's modulus, $\rho$ is density, and $\eta_{i}$ is the intrinsic loss factor. The colours indicate different material classes: ceramics, glasses, silicon and diamond (red); metals (orange); elastomers (blue); and polymers (purple). Dashed lines represent equivalent material bandwidth products. As a general material class, polymers have the highest material bandwidth product, at approximately three orders of magnitude above the traditional AFM cantilever materials silicon and SiN (see Supplementary Information). b. Predicted imaging bandwidth as a function of rectangular cantilever geometry for silicon and SU-8. Allowable geometries: $5 \leq k \leq 100 \mathrm{~N} \mathrm{~m}^{-1}, f_{0} \leq 5 \mathrm{MHz}$, a thickness-to-width ratio between 1:100 and 1:3, and the often used 3:1 length-to-width aspect ratio. For similar cantilever length and width, cantilevers made from SU-8 yield roughly one order of magnitude higher predicted imaging bandwidth.
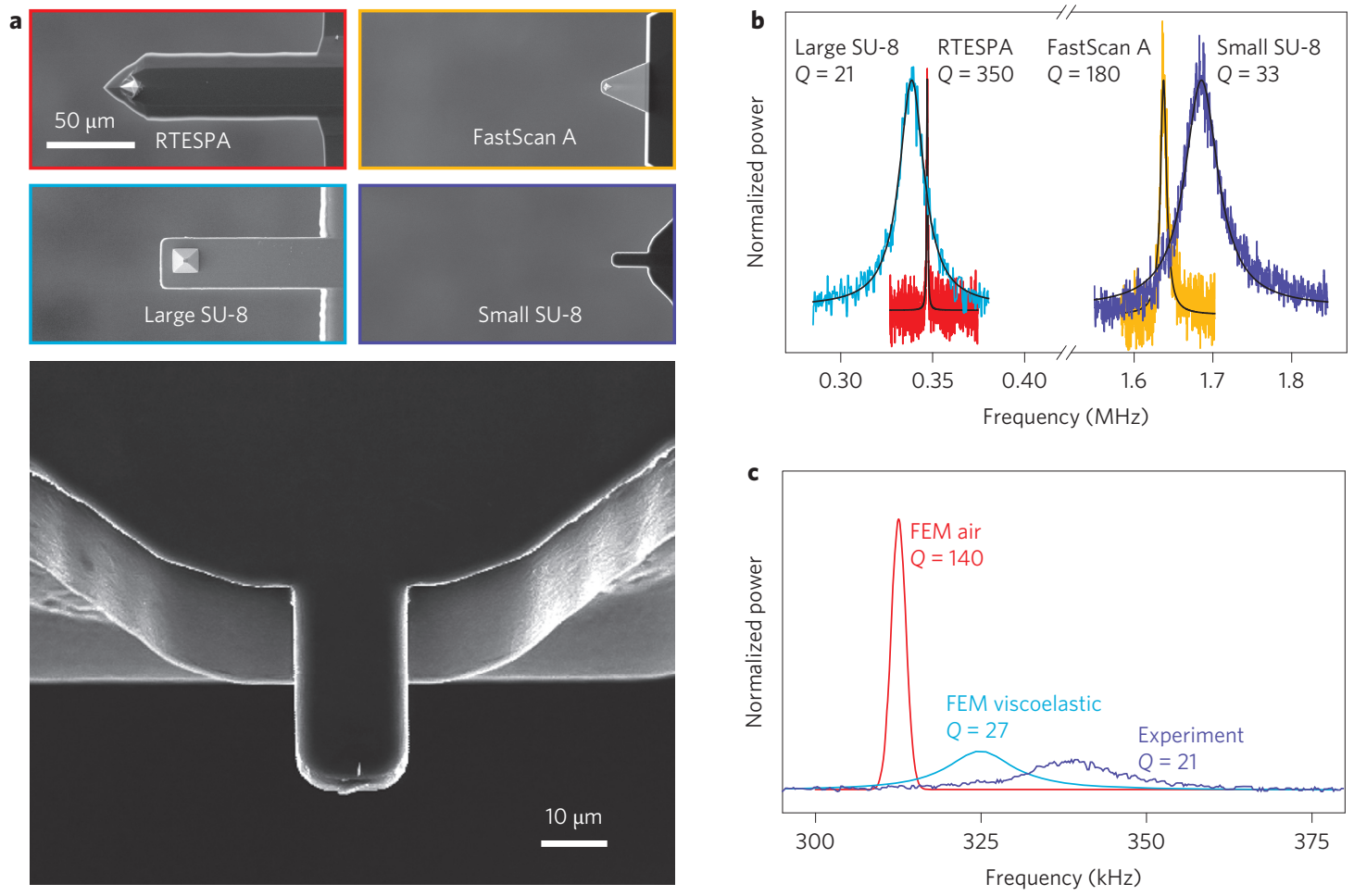

Figure 3 | Polymer cantilevers and their mechanical response. a, Comparison between a large and small commercial AFM cantilever and a large and small SU-8 cantilever (top) and overview image of a small SU-8 cantilever (bottom). b, From the measured thermomechanical noise spectra, the RTESPA and large SU-8 cantilevers have similar $f_{0}$ and $k_{1} f_{0}^{\text {large } \mathrm{Si}}=347 \mathrm{kHz}$ versus $f_{0}^{\text {large SU-8 }}=337 \mathrm{kHz}$ and $k^{\text {largeSi }}=47 \mathrm{~N} \mathrm{~m}^{-1}$ versus $k^{\text {largeSU-8}}=74 \mathrm{~N} \mathrm{~m}^{-1}$ for the large cantilevers and $f_{0}^{\text {small } \operatorname{SiN}}=1,640 \mathrm{kHz}$ versus $f_{0}^{\text {small SU-8 }}=1,690 \mathrm{kHz}$ and $k^{\text {smallsiN }}=9 \mathrm{~N} \mathrm{~m}^{-1}$ versus $k^{\text {smallsU-8 }}=6 \mathrm{~N} \mathrm{~m}^{-1}$ for the small cantilevers), but the $Q$ differ by a factor of 17. Similarly, the small SU-8 and FastScan A cantilevers have values of $Q$ that differ by a factor of 5 . The data are normalized by the fitted peak height. c, Simulated thermal noise spectra of an SU-8 cantilever due solely to air damping (red) or solely to viscoelastic damping (blue curve); thermoelastic damping is negligible (Supplementary Section 4). FEM, finite element modelling. The $Q$ due to viscoelastic damping closely matches the large SU-8 data from $\mathbf{b}$, which is given as a reference (purple curve). The data are normalized by area.

a cantilever with a tapping-in-air bandwidth of $210 \mathrm{kHz}$, a factor of 131 improvement over traditional tapping-in-air cantilevers and a factor 19 improvement over conventional cantilevers of similar size, resonance frequency and spring constant. This tapping-in-air bandwidth matches or exceeds the tapping-in-fluid bandwidth of all but the very smallest HS-AFM cantilevers ${ }^{1}$.

HS-AFM imaging of biological samples has revolutionized the direct observation of fast biomolecular processes with nanometre 

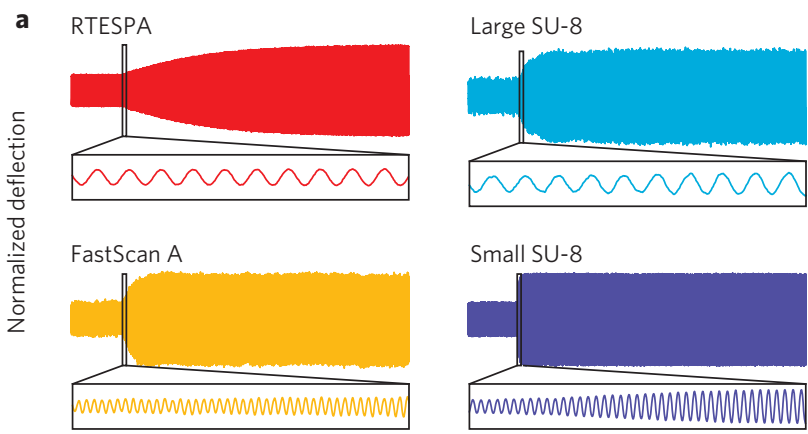

Small SU-8
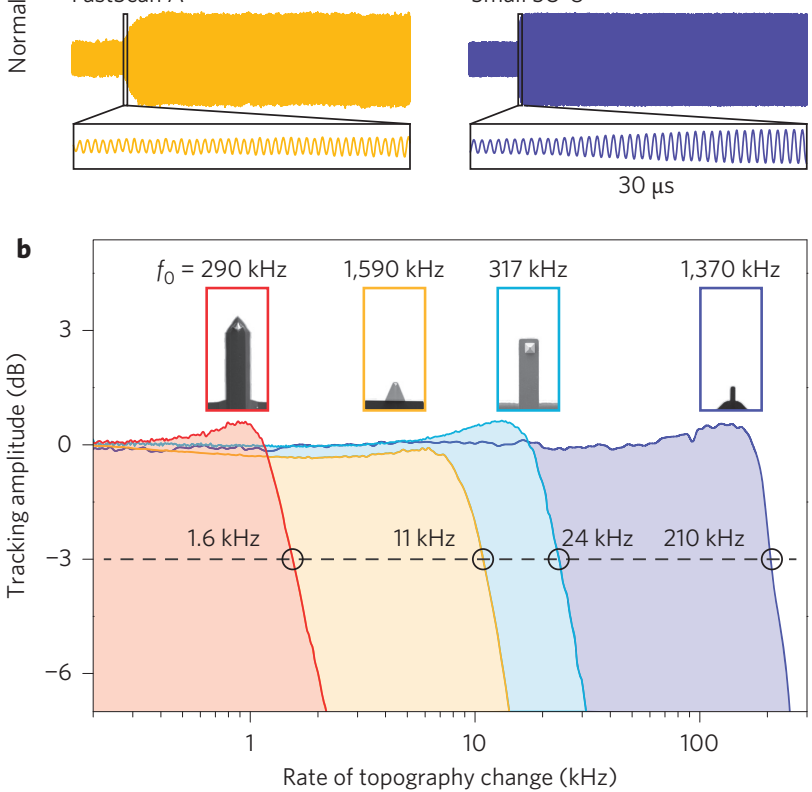
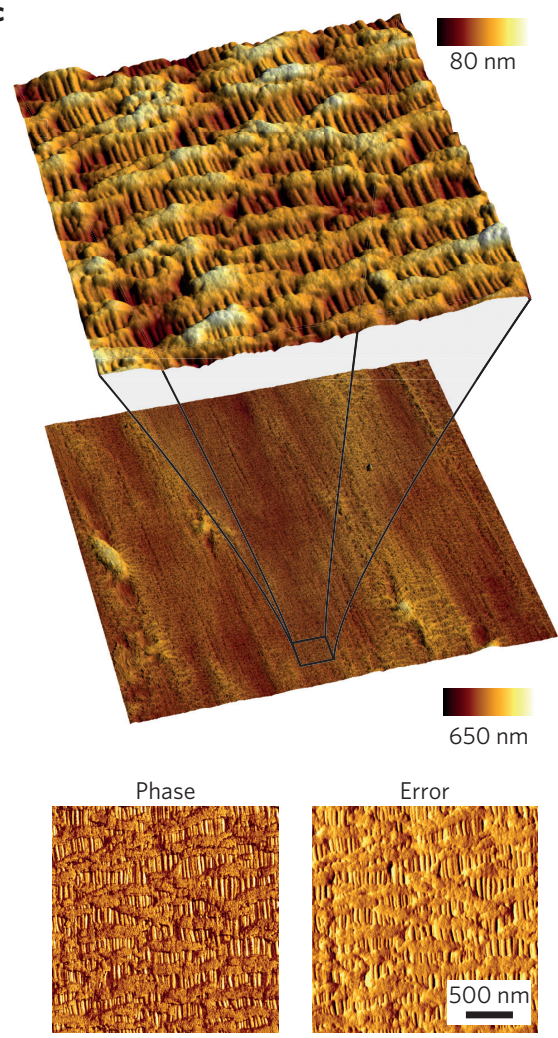

Error

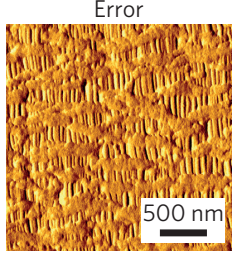

Figure 4 | HS-AFM tapping-mode imaging in air using small SU-8 cantilevers. a, Cantilever amplitude response to a step increase in drive amplitude. The time taken to reach the steady state is related to the ratio $f_{0} / Q$. b. Response of the cantilever amplitude to surface height modulation. The $-3 \mathrm{~dB}$ frequency of the small SU-8 cantilever is increased by a factor of 19 compared with a conventional AFM cantilever of similar size, resonance frequency and spring constant. c, High-resolution overview imaging of a Celgard membrane at a tip surface speed of $261 \mu \mathrm{m} \mathrm{s}{ }^{-1}$, maintaining good tracking. The overview image has dimensions of $8,192 \times 3,200$ pixels and the digital zoom-ins $656 \times 256$ pixels, all in the same region. Error scale: -2 to $2 \mathrm{~nm}$; phase scale: -8 to $8^{\circ}$.

resolution $^{1}$. The need for HS-AFM imaging in air is not as much driven by the desire to image fast processes, but rather by productivity and the need to image larger areas quickly, as is often required in industrial applications for failure analysis. With large scan areas, the surface speed and rate of topography change are high, even at slower scan rates. The use of parallel cantilever arrays is one approach to imaging large areas without increasing the surface speed ${ }^{9}$, but this increases the complexity considerably. With the detection bandwidth we demonstrate here, it becomes practical to collect high-resolution, highly detailed images of large areas using a single cantilever. To demonstrate this, we imaged large areas of a monolayer polypropylene lithium battery separation membrane (Celgard monolayer PP). The difficulty of imaging the freely suspended fibrils in this material have made it a reference sample for assessing AFM imaging speed performance. Using large SU-8 cantilevers we obtained a ten times increase in imaging speed compared with standard cantilevers (Supplementary Section 5). With our small SU- 8 cantilevers we imaged a $30 \times 30 \mu \mathrm{m}^{2}$ area of the Celgard membrane at a surface speed of $261 \mu \mathrm{m} \mathrm{s}^{-1}$, using a standard HS-AFM system (Bruker FastScan). We achieved imaging speeds six times faster than previously reported for this system. At this point we were mainly limited by the AFM controller bandwidth $(30 \mathrm{kHz})$ rather than the cantilever bandwidth $(210 \mathrm{kHz})$. The digital zoom in Fig. $4 \mathrm{c}$ presents a $2.4 \mu \mathrm{m}$ crop of the overall image, corresponding to $656 \times 256$ pixels imaged in an equivalent time of $2 \mathrm{~s}$, showing very well resolved individual fibrils of the Celgard membrane.

The increased imaging speed (Fig. 1c and $4 \mathrm{~b}$ ) clearly shows the enormous potential of engineering the materials properties of cantilevers. We have not yet achieved the $Q$ values of $\sim 2-4$ that are obtained in liquid. However, in liquid, the resonance frequency is also reduced by a factor of 3-5 due to the inertial effects of the surrounding liquid. Methods to actively control $Q$ have previously been proposed ${ }^{10,17}$, although generally to increase, rather than decrease $Q$. These methods could be used to decrease the $Q$ by a small amount ${ }^{17,18}$, which could provide a modest additional increase in the detection bandwidth in parallel with our approach.

In addition to tapping-mode AFM imaging, cantilevers with intrinsically low $Q$-factors would be beneficial for the suppression of unwanted oscillations in many other modes of AFM imaging. Examples are HS-contact mode AFM imaging, where cantilever oscillations may appear as a result of a brief loss of $\operatorname{contact}^{19}$, in force-curve-based imaging modes (after breaking free from adhesion $^{20}$ ), or in nanoscale mechanical property measurements ${ }^{21}$ (to decrease the measurement time). The intrinsically lowered $Q$ may not be suitable for all situations, however. The lower $Q$ fundamentally reduces the cantilever sensitivity to very small forces ${ }^{22}$, such as those being used for submolecular-resolution AFM imaging $^{23}$ or magnetic resonance force microscopy ${ }^{24}$. This reduced sensitivity has raised concerns that low- $Q$ cantilevers would increase sample damage or tip wear. However, most tip/ sample damage occurs at topography transients, where the AFM cannot maintain a constant tip-sample force. Because low- $Q$ cantilevers detect topography faster, the AFM feedback loop can react to transients faster, thereby yielding more consistent tip-sample forces, even at slow imaging rates (Supplementary Section 6). Tip wear, however, is a concern for polymer cantilever tips. Here, we have circumvented this issue by growing a carbon tip on the cantilever. While this process may be automated at the wafer $\mathrm{scale}^{25}$, a method for batch-fabrication of integrated hard tips would be desirable. A 
further concern is long-term stability. However, we used our cantilevers over a period of 1.5 years after fabrication without issue (Supplementary Section 7). Furthermore, Martin and colleagues studied the aging of photoplastic cantilevers and found no change in cantilever bending after 1 year in storage both in cleanroom and uncontrolled laboratory environments ${ }^{26}$.

Fast AFM imaging requires a high bandwidth of all system components. Active cantilevers ${ }^{17}$ and flexure-based scanners ${ }^{5,27}$ can achieve high actuation bandwidths, and high-bandwidth controllers have been developed ${ }^{28,29}$. Nearly two decades after the first reports on small high-speed AFM cantilevers ${ }^{4,5}$, the cantilever bandwidth in air is again the primary speed limitation. The current miniaturization approach towards higher cantilever bandwidths has reached a natural limit. In this work, we have shown how to overcome this roadblock by changing the cantilever material to a polymer with high materials bandwidth product. The internal damping in polymers yields cantilevers with inherently low $Q$, thereby mimicking the fast response of HS-AFM cantilevers in liquid. The achieved detection bandwidth of $210 \mathrm{kHz}$ is an increase of over two orders of magnitude over conventional AFM cantilevers. This enables a whole new generation of HS-AFMs for general-purpose use. Our results using the off-the-shelf polymer SU-8 are only an indication of what could be possible with a material optimized for a high material bandwidth product. Future work will focus on testing different polymers, polymer blends and hybrid structures. Preliminary calculations indicate that such cantilevers could have quality factors in air below 5 with resonance frequencies above $5 \mathrm{MHz}$ (Supplementary Section 8), resulting in detection bandwidths of several megahertz, thereby enabling routine nanocharacterization and nanomanipulation far beyond what is currently possible.

\section{Methods}

Methods and any associated references are available in the online version of the paper.

Received 15 January 2015; accepted 29 September 2015; published online 23 November 2015

\section{References}

1. Kodera, N., Yamamoto, D., Ishikawa, R. \& Ando, T. Video imaging of walking myosin V by high-speed atomic force microscopy. Nature 468, 72-76 (2010).

2. Igarashi, K. et al. Traffic jams reduce hydrolytic efficiency of cellulase on cellulose surface. Science 333, 1279-1282 (2011).

3. Fantner, G. E., Barbero, R. J., Gray, D. S. \& Belcher, A. M. Kinetics of antimicrobial peptide activity measured on individual bacterial cells using highspeed atomic force microscopy. Nature Nanotech. 5, 280-285 (2010).

4. Walters, D. A. et al. Short cantilevers for atomic force microscopy. Rev. Sci. Instrum. 67, 3583-3590 (1996).

5. Ando, T. et al. A high-speed atomic force microscope for studying biological macromolecules. Proc. Natl Acad. Sci. USA 98, 12468-12472 (2001).

6. Li, M., Tang, H. X. \& Roukes, M. L. Ultra-sensitive NEMS-based cantilevers for sensing, scanned probe and very high-frequency applications. Nature Nanotech. 2, 114-120 (2007).

7. Antognozzi, M. et al. A new detection system for extremely small vertically mounted cantilevers. Nanotechnology 19, 384002 (2008).

8. Sanii, B. \& Ashby, P. D. High sensitivity deflection detection of nanowires. Phys. Rev. Lett. 104, 147203 (2010).

9. Minne, S. C. et al. Centimeter scale atomic force microscope imaging and lithography. Appl. Phys. Lett. 73, 1742-1744 (1998).

10. Mertz, J., Marti, O. \& Mlynek, J. Regulation of a microcantilever response by force feedback. Appl. Phys. Lett. 62, 2344-2346 (1993).
11. Sader, J. E. Frequency response of cantilever beams immersed in viscous fluids with applications to the atomic force microscope. J. Appl. Phys. 84, 64-76 (1998).

12. Milhiet, P.-E. et al. Deciphering the structure, growth and assembly of amyloidlike fibrils using high-speed atomic force microscopy. PLoS ONE 5, e13240 (2010).

13. Uchihashi, T., Iino, R., Ando, T. \& Noji, H. High-speed atomic force microscopy reveals rotary catalysis of rotorless F1-ATPase. Science 333, 755-758 (2011).

14. Casuso, I. et al. Characterization of the motion of membrane proteins using high-speed atomic force microscopy. Nature Nanotech. 7, 525-529 (2012).

15. Preiner, J. et al. IgGs are made for walking on bacterial and viral surfaces. Nature Commun. 5, 4394 (2014).

16. Genolet, G. et al. Soft, entirely photoplastic probes for scanning force microscopy. Rev. Sci. Instrum. 70, 2398-2401 (1999).

17. Sulchek, T. et al. High-speed tapping mode imaging with active $Q$ control for atomic force microscopy. Appl. Phys. Lett. 76, 1473 (2000).

18. Sulchek, T., Yaralioglu, G., Quate, C. \& Minne, S. Characterization and optimization of scan speed for tapping-mode atomic force microscopy. Rev. Sci. Instrum. 73, 2928-2936 (2002).

19. Humphris, A. D. L., Miles, M. J. \& Hobbs, J. K. A mechanical microscope: highspeed atomic force microscopy. Appl. Phys. Lett. 86, 034106 (2005).

20. Rosa-Zeiser, A., Weilandt, E., Hild, S. \& Marti, O. The simultaneous measurement of elastic, electrostatic and adhesive properties by scanning force microscopy: pulsed-force mode operation. Meas. Sci. Technol. 8, 1333-1338 (1997).

21. Herruzo, E. T., Perrino, A. P. \& Garcia, R. Fast nanomechanical spectroscopy of soft matter. Nature Commun. 5, 3126 (2014).

22. Smith, D. Limits of force microscopy. Rev. Sci. Instrum. 66, 3191-3195 (1995).

23. Gross, L., Mohn, F., Moll, N., Liljeroth, P. \& Meyer, G. The chemical structure of a molecule resolved by atomic force microscopy. Science 325, 1110-1114 (2009).

24. Rugar, D., Budakian, R., Mamin, H. \& Chui, B. Single spin detection by magnetic resonance force microscopy. Nature 430, 329-332 (2004).

25. Kindt, J. H., Fantner, G. E., Thompson, J. B. \& Hansma, P. K. Automated waferscale fabrication of electron beam deposited tips for atomic force microscopes using pattern recognition. Nanotechnology 15, 1131-1134 (2004).

26. Martin, C. et al. Stress and aging minimization in photoplastic AFM probes. Microelectron. Eng. 86, 1226-1229 (2009).

27. Fantner, G. E. et al. Components for high speed atomic force microscopy. Ultramicroscopy 106, 881-887 (2006).

28. Schitter, G., Allgöwer, F. \& Stemmer, A. A new control strategy for high-speed atomic force microscopy. Nanotechnology 15, 108-114 (2004).

29. Kodera, N., Sakashita, M. \& Ando, T. Dynamic proportional-integral-differential controller for high-speed atomic force microscopy. Rev. Sci. Instrum. 77, 083704 (2006).

\section{Acknowledgements}

The authors thank C. Rashti, P. Odermatt and M. Dukic-Pjanic for their assistance. The authors thank the staff of the Centre of Micronanotechnology (CMi) at EPFL for microfabrication assistance and the Atelier de l'institut de production et robotique at EPFL for the fabrication of research equipment. This work was funded by the European Union's Seventh Framework Programme FP7/2007-2011 under grant agreement 286146 and the European Union's Seventh Framework Programme FP7/2007-2013/ERC grant agreement 307338, and the Swiss National Science Foundation through grants 205321_134786 and 205320_152675.

\section{Author contributions}

J.D.A. designed experiments, built instrumentation, performed experiments, analysed data and wrote the paper. B.W.E. built instrumentation and performed experiments. J.G. performed experiments. J.B. designed experiments and coordinated research. A.N. built instrumentation and performed experiments. G.E.F. designed experiments, coordinated research and wrote the paper.

\section{Additional information}

Supplementary information is available in the online version of the paper. Reprints and permissions information is available online at www.nature.com/reprints. Correspondence and requests for materials should be addressed to G.E.F.

\section{Competing financial interests}

The authors declare no competing financial interests. 


\section{Methods}

Cantilever fabrication. The fabrication process is similar to other reported processes $^{16,30}$. The major cantilever fabrication steps were as follows: optional definition of a tip mould in the support wafer, definition of the cantilever layer, build-up of the chip body, release of the cantilevers from the support wafer, deposition of a reflecting surface on the cantilever topside, and growth of sharp carbon tips. Briefly, pyramidal moulds for the cantilever tips were defined in a silicon support wafer using a SiN mask through photolithography, reactive ion etching and $\mathrm{KOH}$ etching of the wafer topside. A layer of SU-8 was spun onto the support wafer, then exposed and developed to define the cantilever. Successive layers of SU- 8 were spun on and exposed to build up the chip body. All chip layers were then developed simultaneously. A brief immersion in $\mathrm{KOH}$ separated the SU-8 structures from the silicon support wafer. A reflective aluminium coating was evaporated onto the cantilever topside. Finally, amorphous carbon tips were grown using electron beaminduced deposition on the end of the pyramidal SU-8 tip structure. More details are provided in Supplementary Section 9.

To calculate the appropriate cantilever dimensions, we input a Young's modulus of $E=4 \mathrm{GPa}$ and a density of $\rho=1,200 \mathrm{~kg} \mathrm{~m}^{-3}$ for SU-8 into the cantilever spring constant equation $k=E w t^{3} /\left(4 l^{3}\right)$ and resonance frequency $f_{0}=0.1615 t / l^{2} \sqrt{E / \rho}$, where $l, w$ and $t$ are the cantilever length, width and thickness, respectively. The resulting design dimensions of the large SU-8 cantilevers were $l=100 \mu \mathrm{m}, w=30 \mu \mathrm{m}$ and $t=10 \mu \mathrm{m}$, and the design dimensions of the small SU-8 cantilevers were $l=25 \mu \mathrm{m}$, $w=10 \mu \mathrm{m}$ and $t=2.5 \mu \mathrm{m}$

Resonance characterization. The thermomechanical spectra were measured to characterize the resonance behaviour of the cantilevers using a Bruker NanoScope V controller and MultiMode V AFM. The cantilever was brought into contact with a freshly cleaved mica surface to obtain the deflection sensitivity. We then withdrew the cantilever from the surface and measured the thermal power spectrum using the Nanoscope software. The data were fit to a Lorentz distribution to extract $f_{0}, k$ and $Q$.

Visualization of cantilever dynamic response. The cantilevers were oscillated at their resonance frequency using a Bruker NanoScope V controller and MultiMode V AFM with a Signal Access Module III, and with an Agilent 33500B external frequency generator generating the drive signal. The amplitude of the drive was modulated with a square wave at a frequency of $20 \mathrm{~Hz}$. To detect the deflection of the small SiN and SU-8 cantilevers, we used a custom-built AFM head ${ }^{31}$. The deflection signal was collected either with the NanoScope controller or with a Picoscope 5242A external oscilloscope at a sampling rate of at least $6 \mathrm{MHz}$

Cantilever bandwidth measurement. We defined the cantilever detection bandwidth as the $-3 \mathrm{~dB}$ frequency of the cantilever response to a measurement signal. The cantilever response is the amplitude of oscillation and the measurement signal is the change in average tip-sample distance. The ratio $\pi f_{0} / Q$ serves as a convenient estimate of this quantity, but is only equal in the simplified case of a damped harmonic oscillator with a linear tip-sample interaction ${ }^{10}$. We measured the cantilever bandwidth by applying a sinusoidal height modulation at variable frequency to the sample while measuring the resulting modulation of the cantilever oscillation amplitude. An external lock-in amplifier reference signal was added to the $z$ signal of an AFM controller to generate surface modulation of a custom-built $z$ scanner. The AFM controller measured the cantilever amplitude and output the amplitude into the external lock-in amplifier. We used the contact-mode scanner transfer function to subtract out the $z$ scanner dynamics from the tapping-mode measurements. For more details see Supplementary Section 10.

HS-AFM imaging. To image the sanded mica sample, we used a modified NanoScope V controller and MultiMode 8 AFM system. The modifications to the system were as follows: a custom readout head for small cantilevers ${ }^{31}$, a custom scanner using combined tube and stack piezo actuators with an open-loop $-3 \mathrm{~dB}$ $z$ bandwidth of $95 \mathrm{kHz}$ and a usable $x-y$ scan range of $\sim 130 \times 130 \mu \mathrm{m}^{2}$ and $z$ scan range of $\sim 5 \mu \mathrm{m}$, and an online, system-identification-based compensation of the lateral scanner resonances ${ }^{32,33}$. We used a small SU-8 cantilever with $f_{0}=1.37 \mathrm{MHz}$. The images taken at 43 and $83 \mathrm{~Hz}$ line scan rates were $512 \times 128$ pixels, the image at $111 \mathrm{~Hz}$ line scan rate was $384 \times 128$ pixels, and the image at $166 \mathrm{~Hz}$ was $256 \times 128$ pixels. The decreasing pixel density was necessary due to the data rate limit of the AFM controller. The Celgard membrane was imaged with an unmodified Bruker Dimension FastScan AFM system, using a small SU- 8 cantilever with $f_{0}=1.74 \mathrm{MHz}$.

Images were processed using standard procedures implemented either in SPIP (Image Metrology) or Gwyddion. We removed line-by-line offset using a median difference correction, and removed the background tilt and bow using a second- or third-order polynomial, with the background fit to a height range defined by a minimum and maximum threshold. Noise was removed by fast Fourier transform filtering (Fig. 1f). The limits of the colour scale were adjusted. All images intended for comparison were put on the same colour scale. Some images were crops taken from a larger image, and some images were presented as a three-dimensional view based on the height data with a colour scale and lighting overlay.

\section{References}

30. Calleja, M. et al. Highly sensitive polymer-based cantilever-sensors for DNA detection. Ultramicroscopy 105, 215-222 (2005).

31. Adams, J. D. et al. High-speed imaging upgrade for a standard sample scanning atomic force microscope using small cantilevers. Rev. Sci. Instrum. 85, 093702 (2014).

32. Burns, D. J., Youcef-Toumi, K. \& Fantner, G. E. Indirect identification and compensation of lateral scanner resonances in atomic force microscopes. Nanotechnology 22, 315701 (2011).

33. Nievergelt, A. P., Erickson, B. W., Hosseini, N., Adams, J. D. \& Fantner, G. E. Studying biological membranes with extended range high-speed atomic force microscopy. Sci. Rep. 5, 11987 (2015). 